\title{
Hydrological dispersion module of the decision support system RODOS
}

\author{
M. Zheleznyak, R. Heling ${ }^{1}$ and W. Raskob ${ }^{2}$ \\ Institute of Mathematical Machines and Systems, National Academy of Ukraine, \\ Prospect Glushkova 42, 03187 Kiev, Ukraine \\ ${ }^{1}$ NRG-KEMA, The Netherlands \\ ${ }^{2}$ Forschungszentrum Karlsruhe and DTI, Germany
}

\begin{abstract}
The Hydrological Dispersion Module (HDM) of the RODOS (real-time on-line decision support) system for nuclear emergency management in Europe is under development at IMMSP Ukraine; at NRG, The Netherlands; at FZK, Germany; at SPA "TYPHOON", , Russia; at IPEP, Minsk, Belanus, and NCSR Demokritos, Greece. Initial testing and customisation work was performed by NPPRI, Slovakia. The hydrological model chain of RODOS was outlined covering processes, such as runoff of radionuclides from watersheds following deposition from the atmosphere (RETRACE), transport of radionuclides in river systems (1-D model RIVTOX) and the radionuclide behaviour in lakes, reservoirs and estuaries (radioecological box model LAKECO, 2-D model COASTOX, and 3-D model THREETOX). The main aim of HDM is the prediction of the dispersion of radionuclides in aquatic systems following atmospheric fallout. HDM provides also the supplementary possibility to simulate the transport of radionuclide transport in aquatic systems following a direct release of radioactivity into the surface waters. In the RODOS system, the calculation results of HDM are transferred to both the aquatic food chain model (LAKECO) and to the dose module (FDMA) to assess the main exposure pathways. To apply HDM, the set of hydrological models have to be adapted together with the RODOS system taking into account the specific properties of the hydrological systems in the areas of interest
\end{abstract}

\section{INTRODUCTION}

The evaluation of the radiological consequences of accidental releases of radionuclides from various sites demonstrated a significant contribution from contaminated water bodies to the dose of the population. This was clearly demonstrated by various radiological impact studies on releases of radionuclides on the environment. Significant dose contributions via aquatic pathways were shown for releases from Oak Ridge Laboratory (on the Clinch River-Tennessee River basin), for releases from the "Mayak" plant (on the Techa River - Ob River system) and finally for releases from the Chernobyl (on the Dnieper River basin and on the Scandavian lake system via atmospheric fallout). Both the remobilisation of dry and wet deposited radioactivity by long term floods and heavy rain events, and the resuspension of sediments during storm events, have resulted in the long-distance migration of radioactivity affecting uncontaminated areas downstream of the release site. This migration results in a dose to the population due to the irrigation of agricultural areas with river water and due to the use of river water as drinking water. Additionally, the remobilisation of radionuclides from the bottom sediments of lakes and reservoirs caused a delayed transfer of activity to the aquatic environment.

Following the Chernobyl accident, the development of the Hydrological Dispersion Module (HDM) inside the RODOS (real-time on-line decision support) system for nuclear emergency management in Europe [1] has been started [2-6]. The work on model development and customisation is sponsored by the Commission of the European Communities within its Radiation Protection Research Programme. The $\mathrm{HDM}$ was developed within the Working Group 4 of the RODOS project by NRG, The Netherlands; FZK, Germany; IMMSP, Ukraine; and SPA "Typhoon", Russia; IPEP, Belarus; and NCSR "Demokritos", Greece. Initial testing and customisation work was performed by NPPRI, Slovakia. The software framework of the whole RODOS system is being developed at Forschungszentrum, Karlsrube (FZK/KET), which acts as the co-ordinator in the CEC research programme. Since 2001 the RODOS implementation in East- and West-European countries has started as part of national programs of nuclear emergency preparedness and response.

\section{HYDROLOGICAL MODELLING IN RODOS - OBJECTIVES AND FUNCTIONALITY}

The main objectives of the HDM are both the simulation of the radionuclide concentration in various compartments of the hydrosphere following accidental releases from nuclear installations, and to prepare the necessary input data for the estimation of the dose to the public via aquatic exposure pathways. In its standard application, HDM simulates the concentrations of radionuclides in aquatic media following an atmospheric fallout. Moreover, HDM provides also the possibility to consider direct releases of radioactive materials into water bodies. This second function, developed for particular types 
of accidents with nuclear power plants, and provides in addition the possibility for model calibration and tuning on the basis of the previously recorded accidents.

As the first phase after an accident has passed, the Atmospheric Dispersion Module (ADM) of RODOS simulates the transport and dispersion of the radioactive cloud using either numerical weather forecasts data or real-time meteorological data. Furthermore, it calculates the deposition to the various surfaces including the water bodies in the contaminated area. The HDM has to use the atmospheric fallout data from the atmospheric dispersion module (ADM), to consider all aquatic pathways and media and finally prepares the input data for the aquatic food chain and dose module FDMA. Therefore, in the functional specification of HDM, it was defined that the system is able to simulate the radionuclide concentration in water, suspended sediment, bottom sediments and fish in

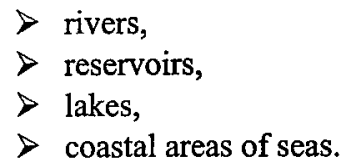

To simulate lateral inflow of radionuclides from contaminated watersheds to these water bodies, also the wash-out of radionuclide had to be modelled. As RODOS is designed to predict the short-term and longterm consequences of accidental releases, also the HDM contain models with these different temporal and spatial scales, ranging from the emergency phase (up to 2-7 days after a release, when real-time and meteorological forecasting data can be used) to the intermediate (up to several months) and long-term. (years) post-accidental phase.

The results of both HDM and FDMA are also used in the RODOS countermeasure subsystem to evaluate the efficiency of possible aquatic countermeasures.

\subsection{Characterisation of transport, dispersion and exchange processes}

Transport, diffusion and exchange processes differ for the various water bodies. Therefore, one has to consider separately three areas: runoff, transport in rivers, and behaviour of radionuclides in lakes and reservoirs. Furthermore it is reasonable to subdivide the major processes into a hydrological part, e.g. water - and suspended sediment transport, and into a radio-chemical part (e.g. sorption to particulate matter)). The basic approach of the RODOS - HDM model system is to provide the user the possibility to simulate both the hydrodynamic and radiological aspects separately for different water bodies and for different temporal and spatial scales.

Specific for radionuclide transport models - in comparison with other water quality models - are the physical- chemical exchange processes in the system "water - suspended sediments - bottom sediments" $[7,8]$.

Mathematical models describing the sorption of metal on homogeneous surfaces, primarily metal hydroxides on reference clay minerals, are based on surface complexation and ion-exchange theories. However, the traditional approach in describing and predicting the fate of radionuclides on heterogeneous solids such as soil, suspended and bottom sediments is mainly empirical and is still based on the use of the parametrisation of simplified adsorption- sorption kinetics, and on the use of the equilibrium distribution coefficients $\mathrm{K}_{d}=\mathrm{C}_{s} / \mathrm{C}$, where $\mathrm{C}_{s}$ is the concentration of the contaminant sorbed on particulate matter, and where $\mathrm{C}$ is the concentration of the dissolved contaminant, both in equilibrium conditions.

The distribution coefficient $\mathrm{K}_{\mathrm{d}}$, which is dependent on liquid and solid phase characteristics, is the integrated result of various physical-chemical processes controlling the retention of radionuclides. This approach assumes a complete sorption equilibrium, which is however seldom the case in natural conditions. Data, both from laboratory experiments and from field measurements, indicate that sorption of radionuclides by clay minerals, soil and sediments, depends on the nature of clay minerals, and that sorption is a kinetically governed process with time scales up to several years. For various reasons, a kinetic approach of the sorption phenomena is therefore necessary. Thus for a realistic modelling of the vertical migration of radionuclides and heavy metals in soil, in bottom deposition and in the aquatic environment, specific knowledge is required, not only about the sorption equilibrium, but also about the sorption kinetics. There is no generic mathematical description of the sorption processes governing the behaviour of metals and radionuclides. Further a detailed study on the sorption kinetics of radionuclides will allow to examine existing concepts in terms of a more fundamental description of the underlying processes.

In general, models of radionuclide transport in rivers/reservoirs do not include the above mentioned kinetics in their complete details. However, a reasonable level of model complexity, which may reflect the main features of the exchange processes -radionuclide transfer in the system "water - suspended sediments - bottom depositions", transition from the non-equilibrium to the equilibrium state, different 
$K_{d}$ values for bottom deposition and suspended sediments, and different rates for adsorption and desorption - can be represented by the so-called " $K_{d}$ - exchange rates" approach, used in many contemporary models, $[7,8]$. This approach could be also expanded to a more complex two-step kinetic model, describing the ${ }^{137} \mathrm{Cs}$ transfer between the exchangeable and the non- exchangeable form (e.g., [9]). Therefore the " $\mathrm{K}_{d}$ - exchange rates" approach was used as basis in nearly all model of the RODOSHDM.

\subsection{Model chain of HDM}

The RODOS-HDM consists of a number of state-of-the-art hydrological dispersion models for the different types of aquatic systems to predict both the short- and the long-term dispersion of radionuclides in the aquatic environment.

Watershed models RETRACE $[2,5]$ simulate, with different temporal and spatial scales, the water -, sediment - and radionuclide inflow into the river net, and into the lakes and reservoirs within a considered watershed. The calculations are based on measurement data on deposited radionuclides and on meteorological data (precipitation rate and temperature) provided by RODOS-ADM. The temporally distributed data on lateral water inflow and on radionuclide concentrations are calculated for the grid nodes of the river net, for the coastlines of large lakes and reservoirs, or for the separate nodes representing small lakes.

The 1-D river model RIVTOX [2, 3, 10] simulates the cross-section-averaged radionuclide concentration in water, suspended sediments and bottom deposition in the nodes of a river channel net. Two variants of the hydraulics module are available: one variant for modelling the water discharge and elevation in a simplified river net, and a second variant for enhanced river modelling, taking into account water works such as sluices and dams in the river system. RIVTOX uses either output of RETRACE to simulate the transport of radionuclides after wash-off from watersheds or can consider direct releases to the water for the simulation of radionuclide transport from point sources. A specific procedure is used for the interpolation of the RIVTOX results - which are in a river grid - to the input grid of the RODOS dose module FDMA. The coupled subsystem RETRACE-RIVTOX has been validated for the Rhine River basin (Fig.1) and the Iliya River in the Chernobyl zone [2, 5]. RIVTOX was validated also on the basis of data on the Dnieper River, the Clinch River- Tennessee River and on the Vah -Dudvah river system [3, $10]$.

The 2D-model COASTOX $[2,4,10]$ simulates the depth-averaged concentration of radionuclides in the nodes of a rectangular grid covering a water body. This grid can cover a part of a river, in the vicinity of the point source, or a large shallow lake or a reservoir. Simulated time-dependent concentrations can be interpolated to the nodes of the RODOS dose grid or can be used after a crosssectional integration as input for RIVTOX to calculate further propagation by the river. COASTOX was applied to evaluate the efficiency of specific hydrological countermeasures on the floodplain of the Pripyat River and for the simulation of radionuclide transport in the Kiev Reservoir [2, 4, 10]. One important step in the validation of COASTOX was the simulation of the dispersion of ${ }^{137} \mathrm{Cs}$ in the Kralova Reservoir, (part of the Vah River) in Slovakia. The radiocaesium was released into the Dudvah River - a small tributary of the Vah River - during an accidental release by the nuclear power plant Bohunice, in June 1989 [10]. COASTOX appeared to be able to predict the location of contaminated spots on the bottom of the reservoir.
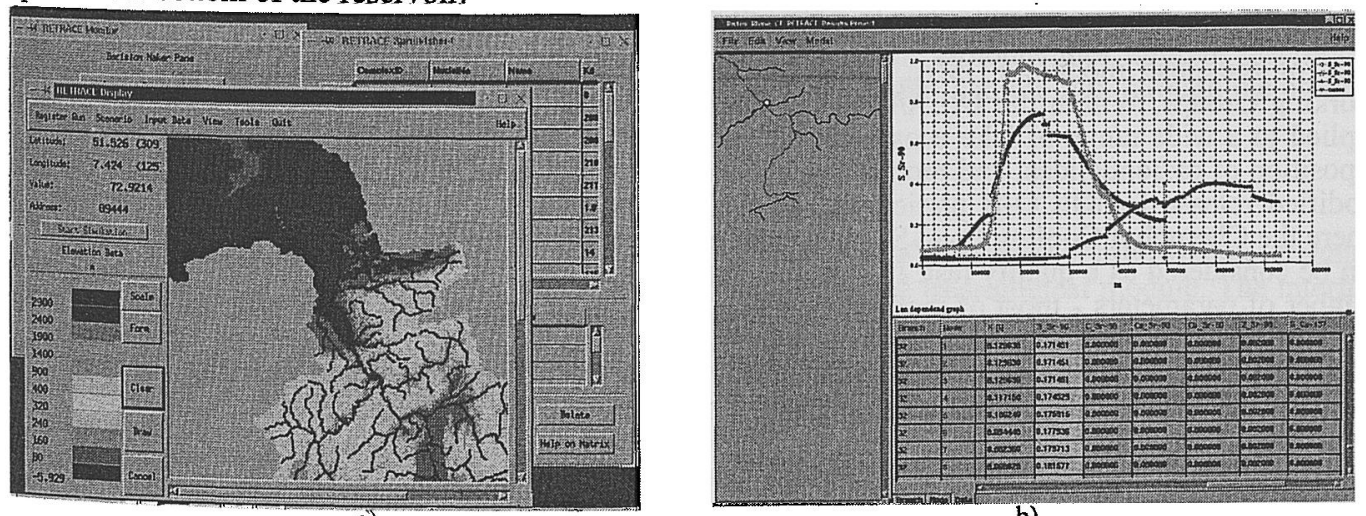

Figure 1: RETRACE (a) and RIVTOX (b) implementation for the Rhine River basin 
The most significant contribution to the contamination of the bottom sediments is the settling of contaminated sediment particles. By means of COASTOX it was predicted revealed that the highest concentration of ${ }^{137} \mathrm{Cs}$ should be found in the central part of the reservoir, in a location where intensive sedimentation, downstream of a steep bottom slope, takes place (Fig. 2). Later field monitoring [10] confirmed this model finding: The maximum ${ }^{137} \mathrm{Cs}$ concentrations in the reservoir, about $35-45 \mathrm{~Bq} / \mathrm{kg}$ in the top sediment layer, and about $60-80 \mathrm{~Bq} / \mathrm{kg}$ in the deeper sediment layer were measured in the same location as predicted by COASTOX, giving a value of about $76 \mathrm{~Bq} / \mathrm{kg}$ (Fig.3) as model result.

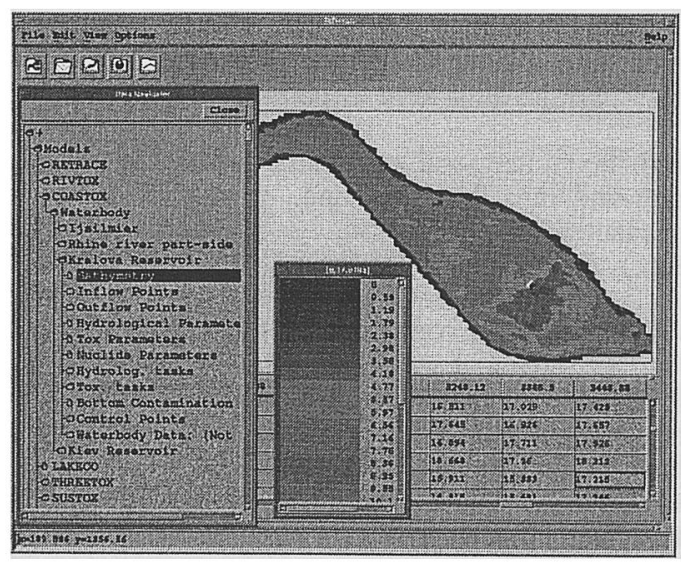

a)

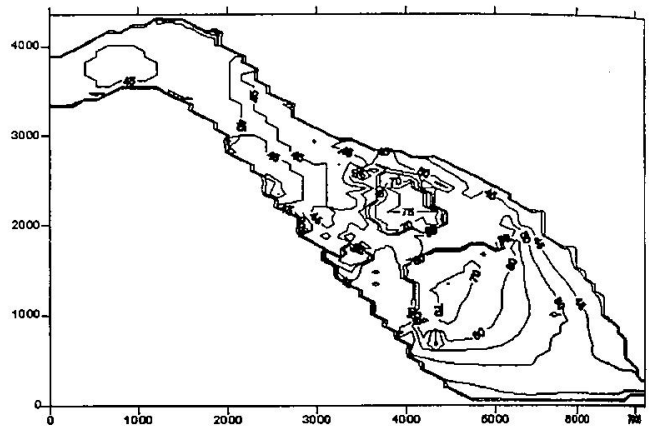

b)

Figure 2: COASTOX implementation for the Kralova Reservoir, Vakh River: a) the bathymetry of reservoir in the HDM interface, b) simulated concentration of ${ }^{137} \mathrm{Cs}$ in upper layer of the bottom sediments $(\mathrm{Bq} / \mathrm{kg})$ after the dispersion of the accidental release in June, 1989.

The 3D model THREETOX [2,7] simulates the radionuclide concentration in nodes of a 3-D grid constructed for deep lakes and other stratified water bodies such as estuaries, cooling ponds of nuclear power plants, and marine or coastal areas. The time-dependent 3-D results are either used to be interpolated to the grid of the dose module FDMA, or can be - in the case of a river outflow from a lake/reservoir - the basis for a boundary condition for the river model RIVTOX. The model has been validated on the radionuclide dispersion in the lake Bodensee (Rhine River Basin, Germany), in the Dnieper-Bug Estuary (Ukraine) and in the Cooling Pond of the Chernobyl Nuclear Power Plant [11] (Fig. 3). THREETOX has been applied in combination with RODOS' marine box-model "POSEIDON" [12] for improvement the transfer coefficients between the coastal boxes of marine model.

A further lake model in HDM, the radioecological box model LAKECO calculates the radionuclide concentration in the biotic and abiotic compartments of the water body [6]. Temporal dynamic of lakeaveraged radionuclide concentrations in water, in suspended sediment, in phytoplankton, fishes and othe: components of the aquatic food chain are simulated. The models results are transferred to the nearest gir node of the dose module FDMA. LAKECO was tested and validated within various international Working Groups. Within the IAEA/CEC_co-ordinated project VAMP, the lake model was successfully applied to a wide range of lake ecosystems in Europe, different in terms of trophic status, climatology, deposition of radionuclides, and morphology. Two versions of LAKECO, the original LAKECO and the modified release LAKECO-B participated in a blind model test within the BIOMOVS II benchmark, where the Chernobyl Cooling Pond Scenario was outlined. Since model tuning was impossible, this study can be considered as a quality test. It appeared that the original LAKECO model, with a relatively greal number of parameters - most of them assessed on the basis of expert judgement, was not able to predic! the concentration in the aquatic system with the required accuracy. However, the enhanced model LAKECO-B gave better model results, which proved that the implementation of new physiologically and physically based sub-models increased indeed the predictive power of the model significantly. 


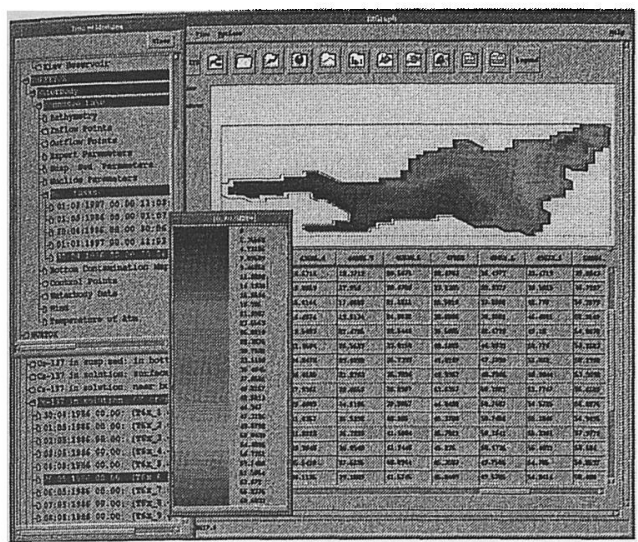

a)

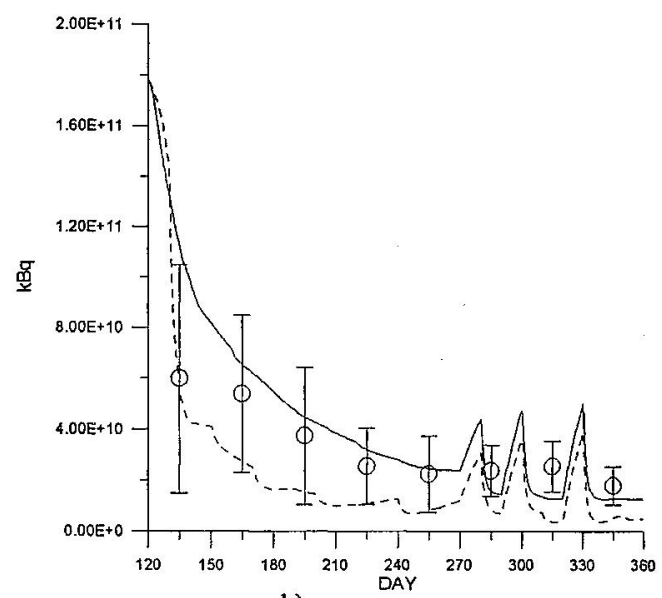

b)

Figure 3: Implementation of THREETOX: a) simulation of ${ }^{137} \mathrm{Cs}$ dispersion in the lake Bodensee (Germany) after the Chernobyl accident, view in THREETOX interface, b) comparison of simulated inventory of ${ }^{137} \mathrm{Cs}$ in Chemobyl Cooling Pond (uncertainty interval of modelling represented by two curves) with the measurement data.

The models RETRACE, RIVTOX, COASTOX, THREETOX and LAKECO establish the main model set of the RODOS HDM. These models are integrated in the HDM interface and cover the main part of required HDM functionality. However within the 4-th Framework Project another set of the modelling tools were developed for special applications [2]. These models were not integrated in the HDM interface, however they can be used as "stand alone" versions for specific cases of the HDM implantation. The one-and-a-half dimension model LATOX simulates the radionuclide concentration averaged over vertical cross-sections of deep stratified lakes in a 1-D temporally variable grid. A special module of LATOX, parametrising the hydrodynamical processes in the lake, provides the simulation of stratified lakes only on the basis of standard meteorological information, without the use od special input data on lake hydro-thermodynamics. The SUSTOX code includes1D-, 2D-, and 3D-models, and is used in HDM to calculate the radionuclide concentration from the unsaturated root zone up to the groundwater. Taking into account the rather low dose significance of groundwater pathways but the high interest of the public, the model can be applied to estimate the efficiency of special late protective countermeasures for groundwater and to simulate the radionuclide flux from groundwater into the river net. Another model, GROWTOX, calculates 1-D or 2-D fields of cross-section or layer-averaged concentrations of radionuclides in groundwater. GROWTOX is useful to estimate radionuclide transport in groundwater on a large scale. To allow a detailed view on the sedimentation and remobilisation processes within a smaller part of a river or reservoir, IPEP, Belarus, has developed the 2-D river model RIVMORPH. This code can also be applied to assess the efficiency of sediment traps in rivers. The infiltration model VADZONE, developed by SPA "Typhoon" describes the fast transfer of radionuclides from the soil surface to the shallow groundwater in the initial phase after an accident. This model allows first assessment of the shallow groundwater which might be contaminated via transport of radionuclides through macropores (e.g. dead roots or rain worm wholes) - a process which is much faster than the flux through the ordinary soil matrix.

\section{HDM INTERFACE}

As the hydrological chain will be an integrated part of the RODOS System, a user friendly graphical interface has been developed to operate the individual models of the hydrological module. The interface provides the possibility of easily accessing all the information necessary to run the individual models as well as displaying the results for the decision makers. The interface allows:

to input and to edit data and parameters through a system of user configured dialogues and input windows;

$>$ to run models separately or simultaneously with the possibility of exchanging data between individual models via shared memory;

to manage the data base and to create predefined scenarios;

to present data base information and on-line results of the simulations in graphs and maps (e.g. contamination); 
to receive data from and transfer data to other RODOS modules (e.g. read results of atmospheric dispersion, forward concentrations to the food chain module).

$>$ to operate individual models via the upper level HDM interface.

Some examples of the graphical user interfaces of main models are presented in Fig.1-3.

Further enhancement of the HDM will include the connection to the marine environment with the integration of the POSEIDON module [12]. In parallel, data assimilation tools will be integrated to allow for the correction of the calculated concentrations based on measurements. With the fully integrated hydrological model chain, it will be possible to describe radionuclide behaviour for releases all over Europe.

\section{Acknowledgements}

The authors are grateful to all those colleagues with whom we worked in the different periods of RODOS-HDM development: A. Popov (SPA "Typhoon" Russia till 2000, now OINPE, Russia), 6 Dontchits, N. Dzhuba, V. Giginyak, S. Kivva, G. Lyashenko, V. Maderich, N. Margvelashvili, A. Marinets, I. Mezhueva, T. Shepeleva, Y. Sorokin, P. Tkalich (IMMSP, Ukraine ), M. Kolomeev, A. Pokhil (SPA "Typhoon", Russia ), A. Andrezhievskiy, A. Trifonov (IPEP, Belarus ), N. Catsaros (NCSR "Demokritos", Greece), O. Slavik (VUJE, Slovakia ) for fruitful input and collaboration.

\section{References}

[1] Ehrhardt J., Brown J., S. French, G.N. Kelly, Mikkelsen T. and Müller H.: Kerntechnik 62 (1997) $122-128$.

[2] Heling R, Raskob W., Popov A. and Zheleznyak M. : Overview of the HDM -Hydrological Dispersion Module of RODOS ( RODOS Report WG4-TN(99)18, Forschungszentrum Karlsruhe, 2000) $128 \mathrm{p}$.

[3] Zheleznyak M., Dontchits G., Dzjuba N., Giginyak V., Lyashenko G., Marinets A., Tkalich P. : RIVTOX - one dimensional model for the simulation of the transport of radionuclides in a network of river channels ( RODOS Report WG4-TN(97)05, Forschungszentrum Karlsruhe, 2000) 48 p.

[4] Zheleznyak M., Shepeleva T. and Mezhueva I. : COASTOX - two-dimensional model describing the lateral-longitudinal distribution of radionuclides in water bodies (RODOS Report WG4-TN(97)07 Forschungszentrum Karlsruhe, 2000) $47 \mathrm{p}$.

[5] Popov A. Documentation of the RODOS External Program RETRACE to Simulate Radionuclide Wash-Off from Watersheds (RODOS Report WG4-TN(99)16 Forschungszentrum Karlsruhe, 2000) $146 \mathrm{p}$.

[6] Heling R. LAKECO-B: model for radionuclide transfer in lakes and biota. (RODOS Report WG4TN(99)17, Forschungszentrum Karlsruhe, 2000) .

[7] Margvelashvili N., Maderich V. and Zheleznyak M., Rad. Prot. Dos. 73 (1997) 177-180.

[8] Onishi Y., Serne J., Arnold E., Cowan C., Thompson F. : Critical review: radionuclide transport, sediment transport, water quality, mathematical modeling and radionuclide adsorption/desorption mechanism (NUREG/CR-1322, Pacific Northwest Laboratory, Richland, 1981) $512 \mathrm{p}$.

[9] Raskob W., Popov A., Zheleznyak M. Heling R., Radioecological Models for Inland Water Systems. (Forschungcentrum Karlsruhe, FZKA 6089, 1998) -225 p.

[10] Slavik O., Zheleznyak M., Dzuba N., Marinets A., Lyashenko G., Papush L., Shepeleva T. and Mihaly B. Rad. Prot. Dos., 73, (1997) 171-175.

[11] Margvelashvili N., Maderich V. and Zheleznyak M., Verification of the THREETOX code within case studies of the lake, estuary and cooling ponds. ( RODOS Report WG4-TN(99)14, Forschungszentrum Karlsruhe, 2000) $47 \mathrm{p}$.

[12] Heling R., Koziy L., Bulgakov, V.: A dynamical approach for the uptake of radionuclides in marine organisms for the POSEIDON-R model system. Proceedings of ECORAD conference 3-7 September 2001, Aix-en-Provence (in press). 